\title{
Detecção de mudanças baseada em objetos para o bairro do Camorim/Jacarepaguá
}

\author{
Object-based change detection for Camorim neighborhood/Jacarepaguá
}

\author{
Gabriel Lousada ${ }^{1}$ \\ Tainá Laeta ${ }^{1}$ \\ Maria Affonso Penna ${ }^{1}$ \\ Manoel do Couto Fernandes ${ }^{1}$
}

${ }^{1}$ Departamento de Geografia, Universidade Federal do Rio de Janeiro - UFRJ. Av. Athos da Silveira Ramos, 274,
Campus Cidade Universitária. Cep21941-916. Rio de Janeiro, RJ, Brasil.
gabriel.lousada@gmail.com, tainalaeta@gmail.com, maria.affonsopenna@gmail.com, manoel.fernandes@ ufrj.com

Recebido em 15/05/2016 - Aceito em 09/12/2016

Received on 15/05/2016 - Accepted on 09/12/2016

\begin{abstract}
RESUMO - A detecção de mudanças baseada em objeto (Object-based Change Detection, OBCD) configura-se como uma área em grande crescimento dentro das pesquisas em Sensoriamento Remoto, isto porque, utilizando-se das possibilidades oferecidas pela análise de imagens baseada em objetos (Geographic Object-based Image Analysis, GEOBIA) é possível produzir mapeamentos de detecção de mudanças em uma única etapa, sem a necessidade da elaboração de mais de um mapa de cobertura da terra para posterior comparação entre as áreas que sofreram alterações. Partindo deste pressuposto, o presente trabalho buscou realizar um mapeamento de detecção de mudanças baseada em objetos para o bairro do Camorim em Jacarepaguá, zona oeste do município do Rio de Janeiro, entre os anos de 2011 e 2015. Os resultados obtidos indicam que 19,12\% da área total do bairro sofreram modificações durante o intervalo de tempo em questão, tal resultado confirma que o bairro vem passando por grandes modificações para construção de infraestrutura dos Jogos Olímpicos de 2016. O processo de validação do mapeamento resultou em uma exatidão global de 0,80 e um índice Kappa de 0,60, considerado de boa qualidade para este tipo de mapeamento, especialmente se levado em conta sua replicabilidade para outras áreas.
\end{abstract}

Palavras-chave: Detecção de mudanças baseada em objetos, GEOBIA, eCognition 9.01 RapidEye, Jacarepaguá.

\begin{abstract}
The Object-based Change Detection (OBCD) is one of the major growing areas within Remote Sensing research, this because, using the possibilities offered by Geographic Object-based Image Analysis (GEOBIA) it is possible to produce a change detection mapping in a single step, without the need to produce more than one land cover map for comparison between areas that have changed. Under this assumption, the present study attempts to make an Object-based Change Detection mapping of Camorim neighborhood, located in Jacarepaguá, west side of the municipality of Rio de Janeiro, between the years 2011 and 2015. The results indicate that 19,12\% of the total district area underwent changes during the timeframe of the research, this result confirms that the neighborhood has been undergoing major changes in infrastructure to host the Olympic Games in 2016. The validation process resulted in an overall accuracy 0.80 and Kappa index of 0.60 , considered good for this type of mapping, especially if taken into account its replicability to other areas.
\end{abstract}

Keywords: Object-based Change Detection, GEOBIA, eCognition 9.01 RapidEye, Jacarepaguá.

\section{INTRODUÇÃO}

Desde o surgimento dos satélites imageadores, a busca por estratégias que permitam o monitoramento de mudanças na superfície terrestre tem sido uma área que recebeu especial atenção dos pesquisadores ligados às ciências ambientais. Este ramo, conhecido como Detecção de Mudanças por Sensoriamento Remoto, passa a ganhar especial interesse com o crescente volume de imagens disponibilizadas gratuitamente, assim como, o advento dos sensores de alta resolução espacial, espectral e temporal.

Singh (1989) define a detecção de mudanças como "o processo de identificar diferenças no estado de um objeto ou fenômeno observando-o em diferentes épocas". A premissa básica desta detecção de mudanças usando Sensoriamento Remoto definida por Singh (1989) baseia-se na ideia de que mudanças na cobertura terrestre resultam em mudanças nos valores de radiância detectados pelos sensores orbitais.

De acordo com Chen et al. (2012), a detecção de mudanças através do Sensoriamento Remoto foi tradicionalmente trabalhada utilizando a abordagem pixela-pixel, no entanto, com o surgimento de sistemas computacionais de alto desempenho, o incremento das possibilidades de processamento permitiu a utilização de segmentações e extração de feições de dados originários de sensores com diversas resoluções espaciais e 
espectrais, assim como, a integração de dados de diferentes datas (resolução temporal). Estes desenvolvimentos possibilitaram a implementação de uma nova abordagem para a detecção de mudanças, a detecção de mudanças baseada em objetos (Object-based Change Detection, OBCD). A detecção de mudança baseada em objetos se configura como um ramo de uma área em exponencial crescimento dentro do Sensoriamento Remoto, a análise de imagens baseada em objetos (Geographic Object-based Image Analysis, GEOBIA).

Um problema frequente na abordagem pixel-apixel para detecção de mudanças é referente à possibilidade de classificações equivocadas de mudanças motivadas pelas diferentes classificações de cobertura da terra em diferentes datas e que são necessárias para determinar as regiões que sofreram mudanças. Utilizando a abordagem baseada em objetos e partindo da premissa proposta por Singh (1989) de que regiões que passaram por mudanças em sua cobertura terrestre apresentam diferentes valores de radiância, Desclée et al. (2006) propõe uma metodologia utilizando uma única classificação que seja capaz de identificar regiões que sofreram alterações em sua cobertura.

A metodologia proposta por Desclée et al. (2006) consiste na segmentação única de duas ou mais imagens, de maneira que as regiões que possuam resposta espectrotemporal semelhantes sejam agrupadas em objetos únicos, assim como, regiões que possuam resposta espectrotemporal diferentes sejam agrupadas em outros objetos. Este tipo de abordagem possibilita que imagens de diferentes datas sejam analisadas como um sistema único passível de mudanças, permitindo sua classificação em uma etapa apenas. Após o agrupamento em objetos das regiões que sofreram e que não sofreram mudanças, é possível realizar a modelagem destas duas classes a partir da amplitude de valores tanto das bandas espectrais das imagens, como através de índices originados das mesmas (ex.: NDVI, NDBI, NDWI, etc.).

Uma das grandes vantagens da análise multitemporal baseada em objetos é a sua replicabilidade para diversas áreas ou cenas de variados sensores, reduzindo a influência de limiares de classificação para determinação de tipos de cobertura terrestre. Este tipo de abordagem tem se mostrado muito efetiva para detecção de mudanças em áreas urbanas, como pode ser observado em Schneider (2012) e Song et al. (2016).

A partir do exposto acima, este trabalho propôs realizar um mapeamento de Detecção de Mudanças baseada em objetos para o bairro do Camorim em Jacarepaguá, zona oeste do município do Rio de Janeiro. A metodologia escolhida baseia-se na proposta de Desclée et al. (2006), e utiliza duas cenas do sensor RapidEye para os anos de 2011 e 2015.

\section{2. ÁREA DE ESTUDO}

A partir da década de 1950 a cidade do Rio de Janeiro passou a sentir os impactos da "febre viária". E é nesse sentido, que ocorre a elaboração de novos planos urbanísticos com o objetivo de construção de novos caminhos na ocupação da cidade, acarretando em um remodelamento da mesma (Abreu, 2006).

Nesse contexto, áreas até então não ocupadas da cidade passam a sofrer grande pressão de ocupação. A expansão urbana da baixada de Jacarepaguá é oriunda da expansão da cidade em direção a São Conrado e a Barra da Tijuca, e a construção viária da Auto-Estrada LagoaBarra. Apesar dessa nova área de expansão da cidade não apresentar o mesmo grau de ocupação das tradicionais áreas da cidade do Rio de Janeiro, o Estado junto com o capital privado vem investindo maciçamente na viabilização de ocupação através da abertura de estradas e ruas, pavimentação, iluminação pública, rede de esgoto, sendo feito com a perda cada vez maior de investimentos públicos em lugares mais necessitados como as zonas urbanas periféricas da cidade.

A baixada de Jacarepaguá (Fig.1) se localiza na cidade do Rio de Janeiro, na região sudeste do Brasil, confinada entre os maciços da Tijuca e da Pedra Branca e ao sul se encontra limitada pelo oceano Atlântico, o que confere uma particularidade em relação ao seu sítio que interfere na circulação atmosférica local. O bairro do Camorim está situado dentro dos limites da baixada de Jacarepaguá, mais especificamente na Área de Planejamento 4 e na XXIV Região Administrativa (Barra da Tijuca) (IPP, 2003).

O bairro do Camorim vem sofrendo um aumento populacional significativo nas últimas décadas, contrastando com a redução de sua área verde. No último decênio, sua população acresceu em 39,90\%, onde em 2000 contava com uma população 786 habitantes, e já no último censo demográfico contava com um total populacional de 1.970 (IBGE, 2010). Vale destacar que nesse mesmo período a cidade do Rio de Janeiro teve um aumento populacional de 7,32\%, passando de 5.857.904 para 6.320.446.

Esse aumento populacional significativo está relacionado ao processo de ocupação da cidade dos anos de 1950, com maior ênfase na baixada de Jacarepaguá e consequentemente o bairro do Camorim a partir da década de 1970 com a implantação do Plano Piloto do arquiteto Lúcio Costa (SUDEBAR, 1977).

Nos últimos anos podemos destacar as crescentes alterações realizadas no bairro, principalmente a partir do final dos anos 1990 com a realização de grandes eventos. Dentre esses eventos, pode-se destacar o Rock in Rio em 2001, os jogos Pan-Americanos em 2007 e mais diretamente dentro do limite do bairro a construção de um conjunto de prédios residenciais que servirá como Vila Olímpica para os Jogos Olímpicos de 2016. 


\section{Bairro do Camorim/Jacarepaguá}

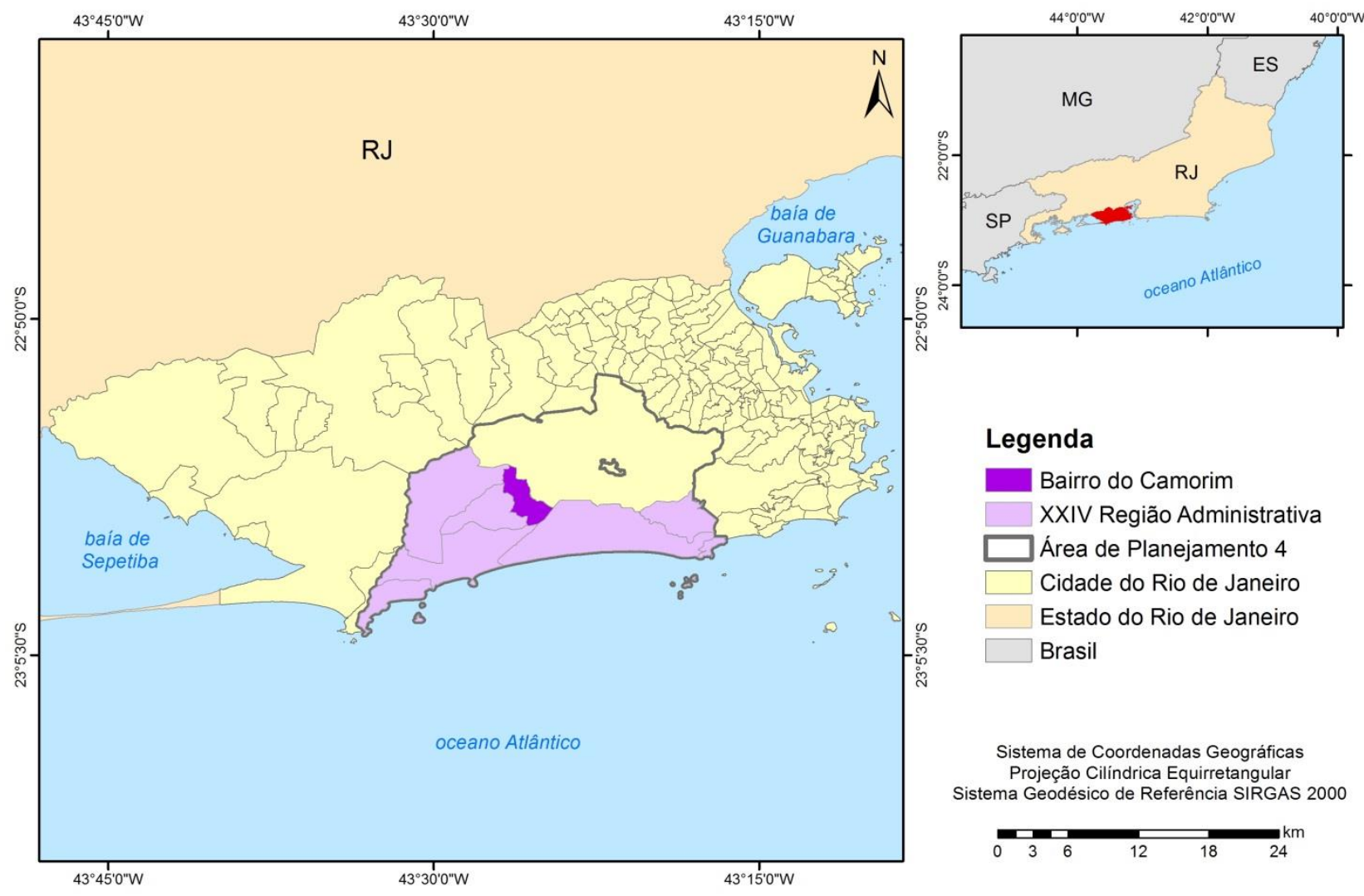

Figura 1 - Localização do bairro do Camorim/Jacarepaguá.

\section{METODOLOGIA}

O sensor RapidEye foi escolhido para realizar a detecção de mudança no bairro do Camorim, isto porque, além de apresentar uma boa resolução espacial e ser disponibilizada gratuitamente pelo Ministério do Meio Ambiente, estas imagens também abrangem o intervalo temporal de interesse do presente trabalho. As imagens utilizadas foram adquiridas pelo satélite em abril de 2011 e janeiro de 2015 (Fig. 2).

Para realização do trabalho a metodologia foi dividida em três etapas: pré-processamento; detecção de mudanças baseada em objetos; e validação.

\subsection{Pré-Processamento}

Duas etapas de pré-processamento foram necessárias para a realização do presente trabalho. Foram elas: correção atmosférica e a normalização radiométrica entre as duas datas.

A correção atmosférica das imagens foi executada no software Erdas Imagine2014, através de sua extensão Atcor 2. Esta extensão permite a retirada dos efeitos atmosféricos da imagem através da utilização de um arquivo de calibração específico para o satélite, além de informações de inclinação solar e inclinação do sensor obtidas nos metadados da imagem, assim como, um modelo de simulação da atmosfera.

A outra etapa do pré-processamento necessária foi a normalização de uma das imagens, isto devido a necessidade de trabalhar com cenas adquiridas em diferentes datas, mesmo com a correção atmosférica, por alterações na calibração do próprio sensor ou por influencias naturais (mudanças de estação), a resposta espectral de um mesmo alvo poderia se apresentar diferenciada nas duas imagens.

Para realizar a normalização é necessária à escolha de uma imagem como referência, e a outra imagem possuirá seus valores modificados em relação a essa. A imagem escolhida como referência foi a do ano de 2011.

O procedimento de normalização entre duas datas demanda a aquisição de pontos invariantes, ou seja, pontos marcados em regiões que não sofreram alterações. Esses pontos foram coletados no software ArcGIS 10.1, totalizando 121 pontos e que contemplassem todas as possíveis classes em um mapeamento de cobertura da terra. Posteriormente foi montado um script no software estatístico R para normalização da imagem de 2015 em relação à de 2011 utilizando os pontos invariantes coletados. 

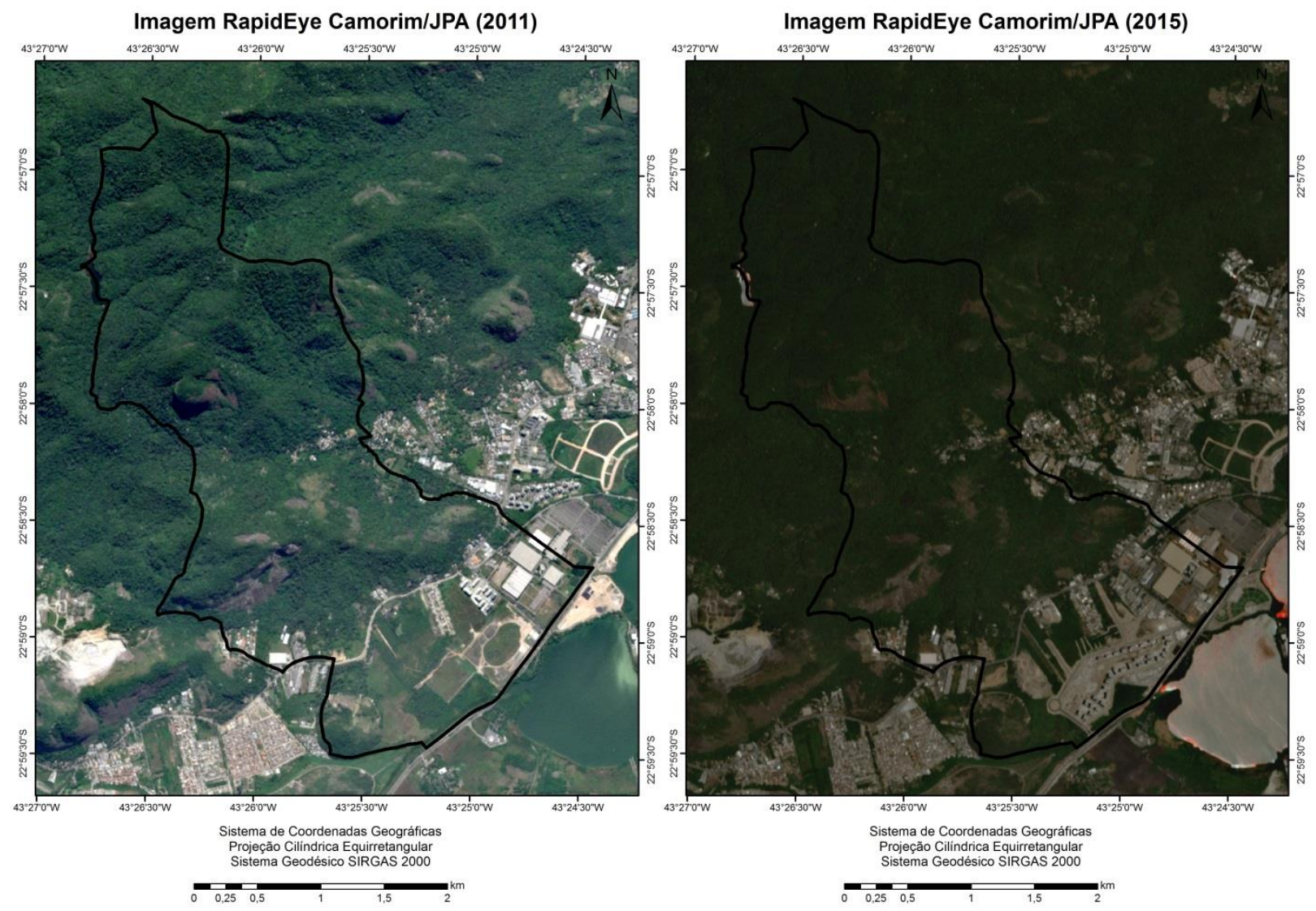

Figura 2 - Bairro do Camorim nas imagens RapidEye 2011 e 2015.

\subsection{Deteç̧ão De Mudanças Baseada Em Objetos}

$\mathrm{O}$ processo de classificação foi realizado no software eCognition 9.01, onde foram inseridos como dados de entrada as imagens RapidEye de 2011 e 2015 pré-processadas.

\subsubsection{Segmentação}

A primeira etapa da classificação realizada foi a segmentação através do algoritmo Multiresolution Segmentation, onde foram realizadas segmentações com dois parâmetros de escala com a finalidade de avaliar qual atenderia melhor as necessidades do mapeamento. Inicialmente foi realizada uma segmentação com o parâmetro de escala 50 (L50), e a partir deste, foi gerada uma nova segmentação em um nível acima com parâmetro de escala 100 (L100). Os outros parâmetros que compõem o algoritmo para segmentação são os aspectos de forma e compacidade, nos quais se utilizou os valores de 0.1 e 0.5 , respectivamente.

Após uma avaliação preliminar foi concluído que a segmentação L100 não alcançava uma delimitação satisfatória, visto que as áreas que sofreram alterações não se apresentavam bem delimitadas pelos segmentos. Sendo assim, a segmentação utilizada foi a L50 (Fig. 3a e 3b).

\subsubsection{Modelagem}

$\mathrm{Na}$ segunda etapa, após o processo de segmentação, foram criadas as classes a serem trabalhadas, sendo elas as classes de mudança e nãomudança, onde o processo subsequente foi a coleta de amostras para as duas classes em todas as áreas internas aos limites do bairro.

Em seguida ao processo de segmentação e antes do processo de classificação, foi feita a criação dos índices que foram utilizados para a modelagem do conhecimento. No trabalho foram criados os índices de normalização da água, área construída, e de vegetação, sendo respectivamente, NDWI (Índice de Água por Diferença Normalizada), NDBI (Índice de Construção por Diferença Normalizada) e NDVI (Índice de Vegetação por Diferença Normalizada). 


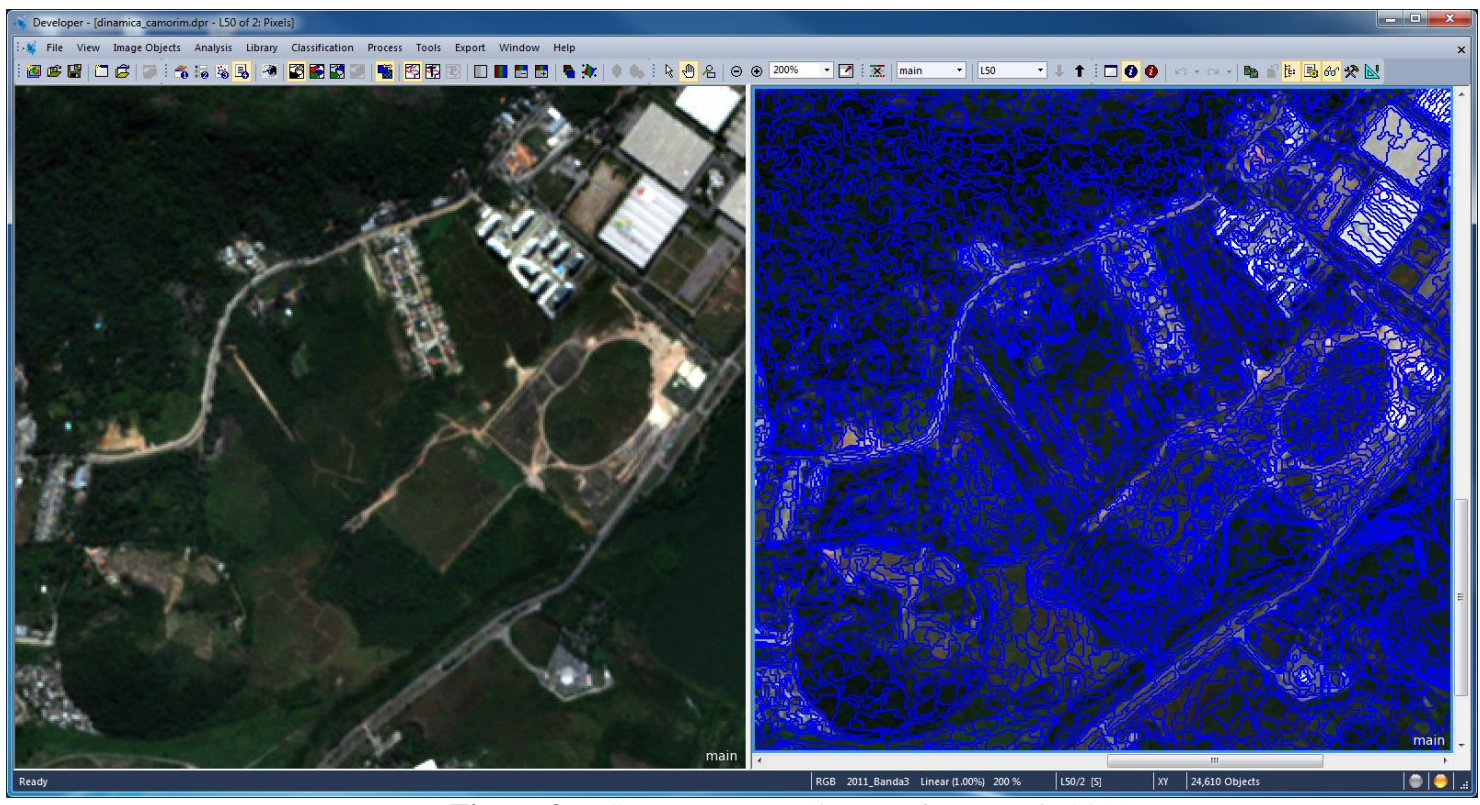

Figura 3a - Segmentação L50 para a imagem de 2011.

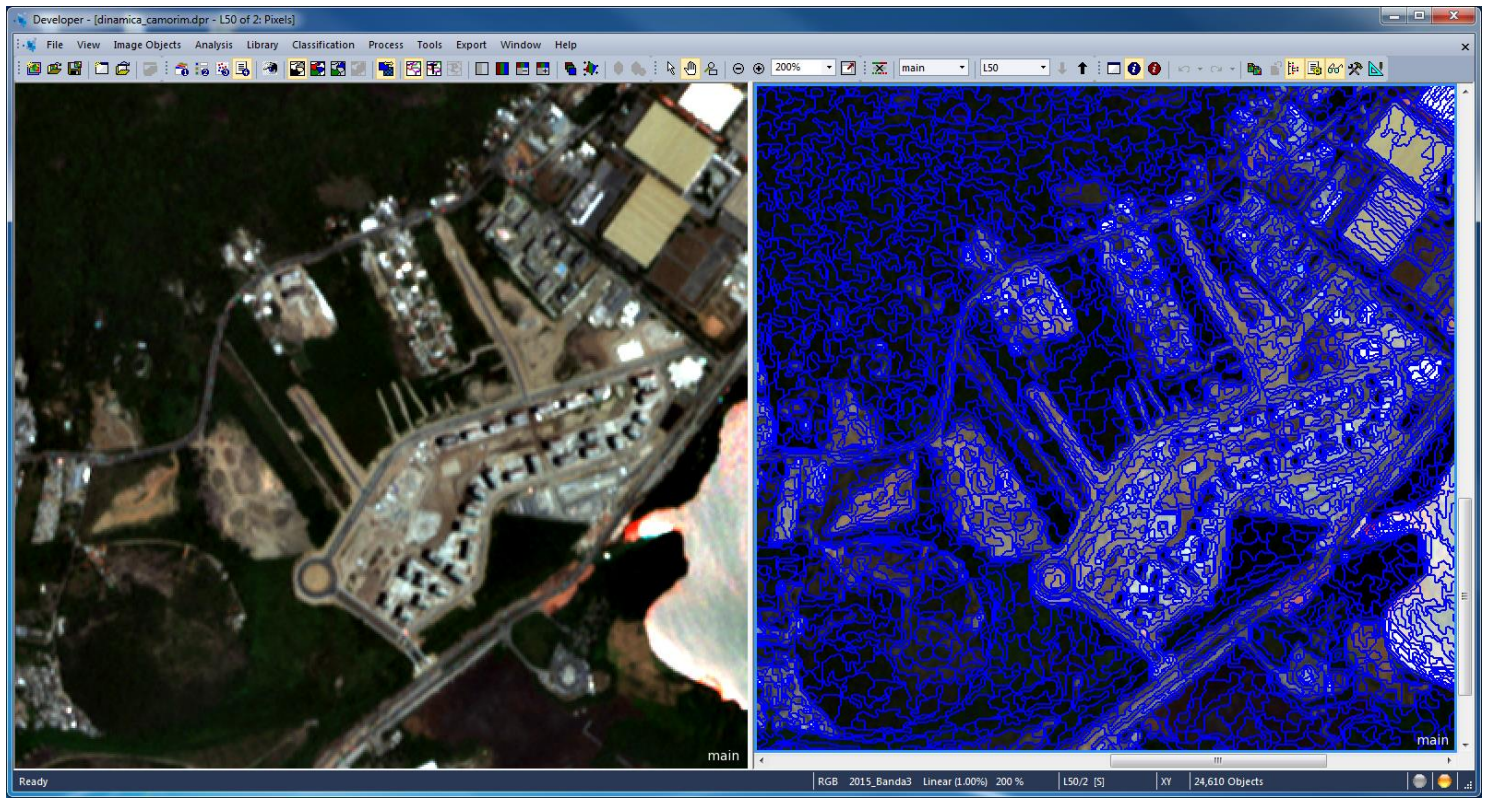

Figura 3b - Segmentação L50 para a imagem de 2015.

Depois da criação desses índices, foram testados descritores de forma a combinar os índices previamente criados, como por exemplo, a razão entre o NDVI de 2011 e o NDVI de 2015, a diferença entre o NDVI de 2011 e de 2015. Os mesmos descritores foram feitos para o NDWI e NDBI. No entanto, durante o processo de modelagem foi observado que o descritor mais adequado para a classificação de detecção de mudança foi a diferença do NDVI, pois todos os outros descritores não conseguiram apresentar um grau de separação suficientemente claro das amostras para realização da modelagem (Fig.4).

Durante o processo de modelagem também foi constatada maior facilidade para a modelagem das amostras referentes às regiões de mudança, isto porque, as áreas de não mudança apresentavam grandes variações em sua composição, principalmente nas regiões florestadas. Por este motivo foi tomada a decisão de modelar apenas as áreas de mudança e através de uma expressão no software, classificar todas as outras áreas como não mudança. 


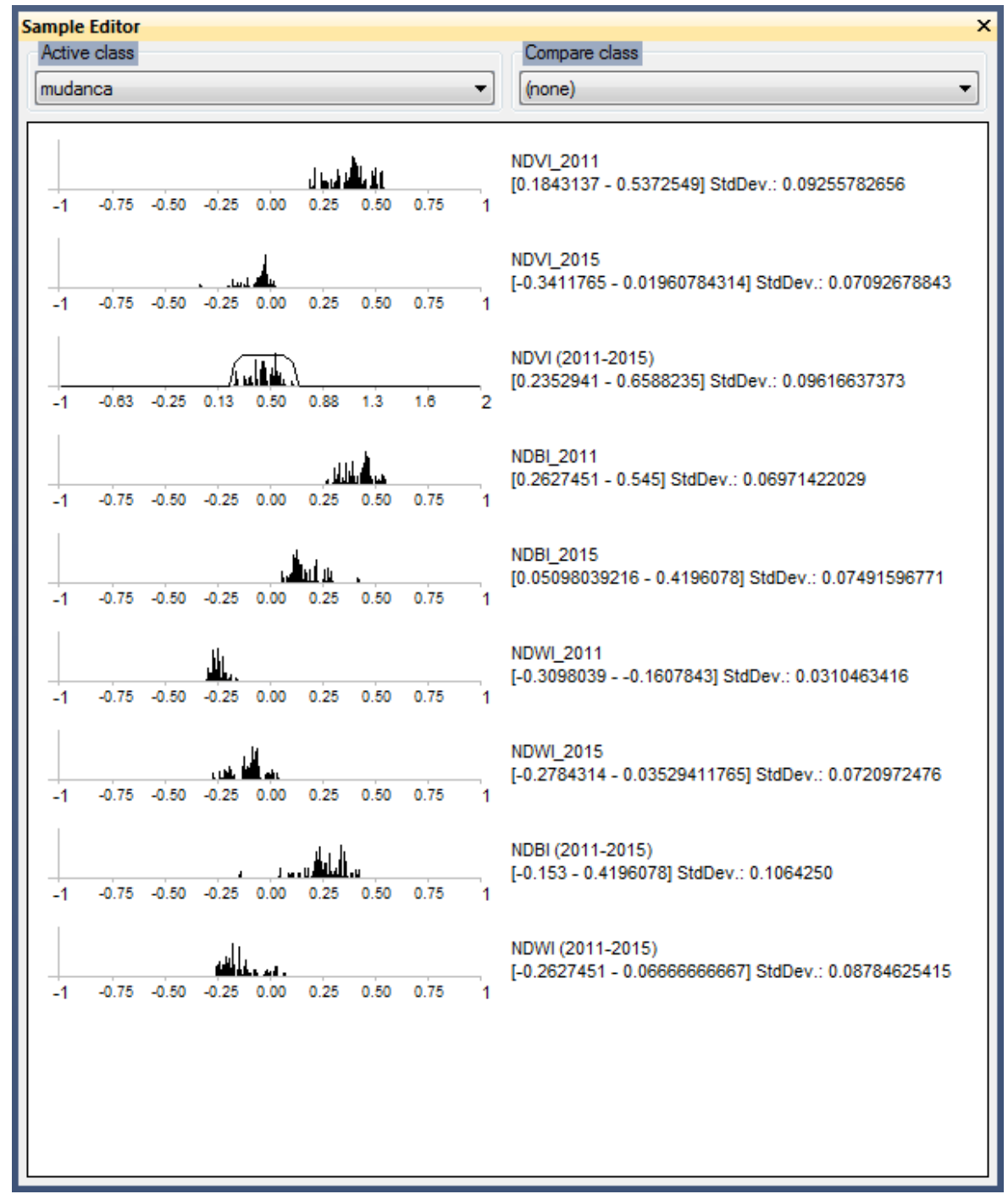

Figura 4 - Processo de modelagem.

\subsubsection{Classificação}

Depois do processo de modelagem da classe mudança, a classificação pode ser realizada de maneira a produzir resultados entendidos como satisfatórios. É importante destacar que a classe mudança adotada neste mapeamento considera não apenas regiões que eram constituídas por floresta e sofreram supressão de mata, mas também podem incluir áreas de regeneração florestal.

Uma questão que se mostrou um entrave metodológico e até o momento da produção deste mapeamento não foi superada, consiste na diferenciação entre áreas que já se apresentavam como solo exposto (como estradas, por exemplo) no ano de 2011 e transformaram-se em canteiros de obra. Apesar de através de uma análise visual este tipo de caso ser de fácil diferenciação, a utilização dos descritores não possibilitou que tal resultado fosse atingido.

Outra área em que se apresentou como uma dificuldade durante a realização do mapeamento é relativa às regiões de afloramento rochoso. Estas regiões apresentaram-se cercadas por vegetação e estavam, localizadas em áreas de elevada declividade, o que acarretou um aumento na incidência de sombra e posterior confusão com áreas de vegetação mais densa. Outro problema foi relativo à presença de vegetação rupestre sobre estes afloramentos, vegetação esta que produziu uma resposta espectral diferenciada para os afloramentos nos dois anos avaliados. Um exemplo de confusão entre mudança e não mudança nas regiões de afloramento rochoso pode ser observado na Fig. 5. 

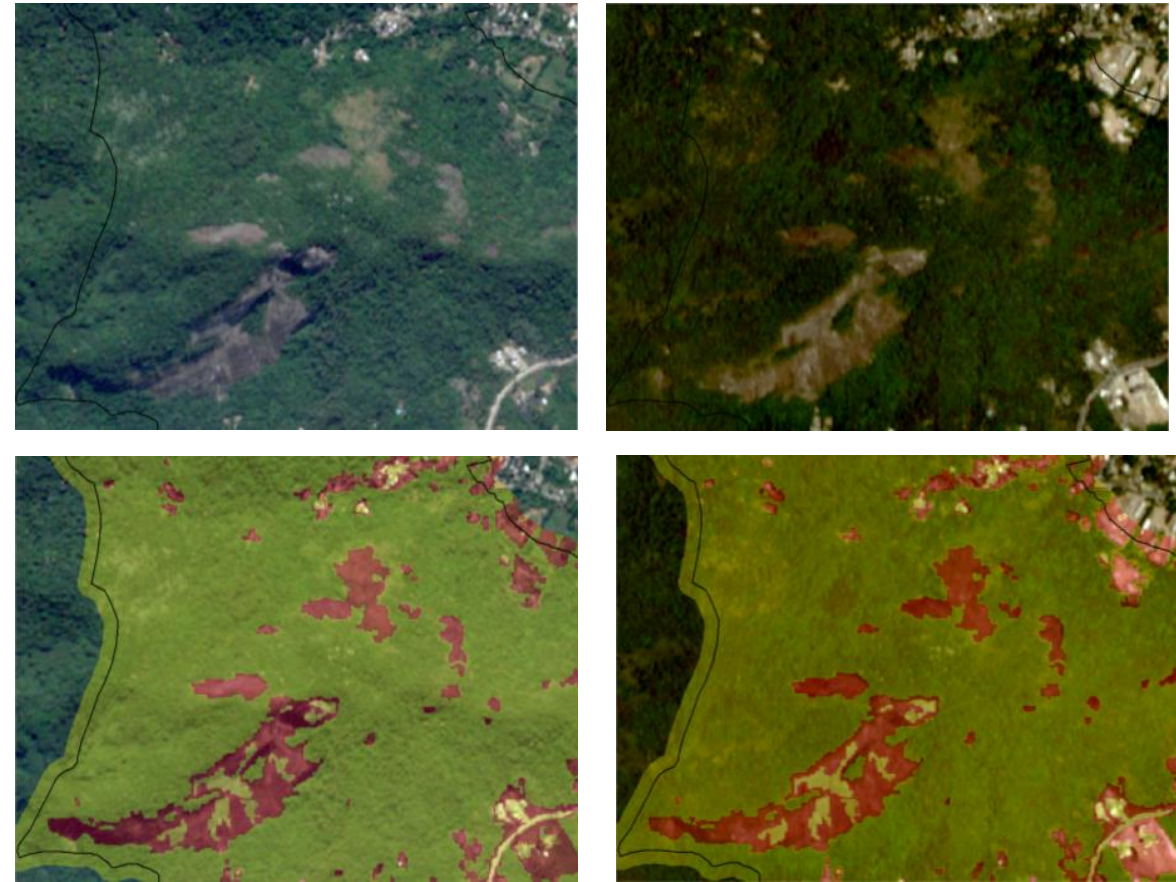

Figura 5 - Regiões de afloramento rochoso e a confusão entre as classes (Cena de 2011 à esquerda e 2015 à direita).

Na porção direita da mesma figura é possível perceber que a modelagem se mostrou adequada para a detecção de mudanças em áreas em que houve a supressão de mata, classificando não só a mudança entre floresta e uma área construída, como entre floresta e vegetação rasteira. Outros exemplos da detecção de mudança podem ser observados na Fig. 6 onde é dada ênfase à região em que está sendo construída a Vila Olímpica.
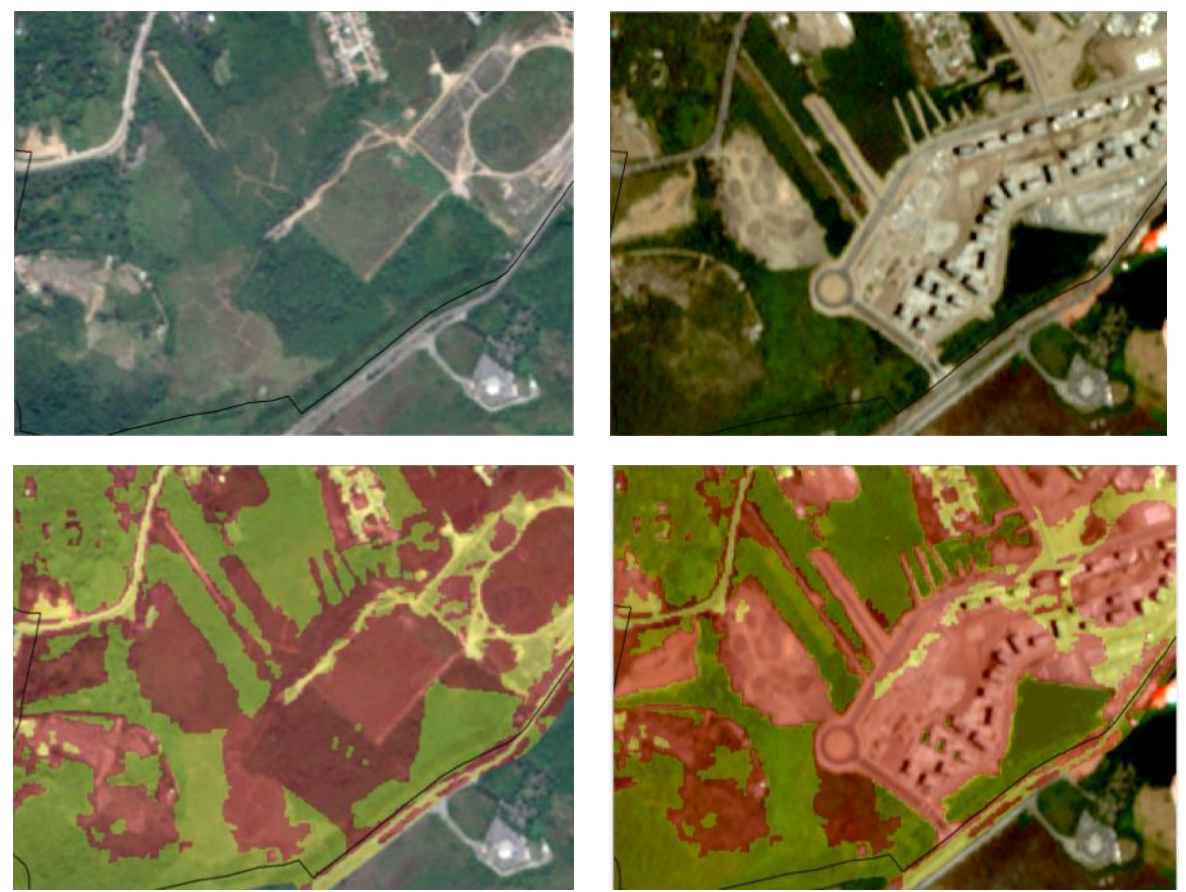

Figura 6 - Detecção de mudanças no canteiro de obras da Vila Olímpica.

\section{RESULTADOS}

\subsection{Edições e Mapa Final}

Por trabalhar com um sensor que possui a resolução espacial de cinco metros, o resultado da classificação possuía uma série de regiões que se encontravam incompatíveis para a escala de mapeamento do trabalho. Ao realizar uma consulta bibliográfica (Antunes \& Siqueira, 2013) foi possível constatar que o sensor RapidEye atende a mapeamentos na escala 1:20.000, podendo ser enquadrados como classe A segundo o Padrão de Exatidão Cartográfica (PEC). Partindo desta informação a área mínima de mapeamento foi definida como $100 \mathrm{~m}^{2}$.

Para que todos os polígonos gerados atendessem a este critério de área mínima de $100 \mathrm{~m}^{2}$ foi necessário realizar uma edição manual através do software ArcGIS 
10.1, onde todo polígono que possuía uma área inferior a área mínima estipulada foi reclassificado com a classe preponderante em seu entorno. Um exemplo deste tipo de edição pode ser observado na Fig.7. Ao todo foram editados manualmente 38 polígonos que possuíam a área inferior a $100 \mathrm{~m}^{2}$.

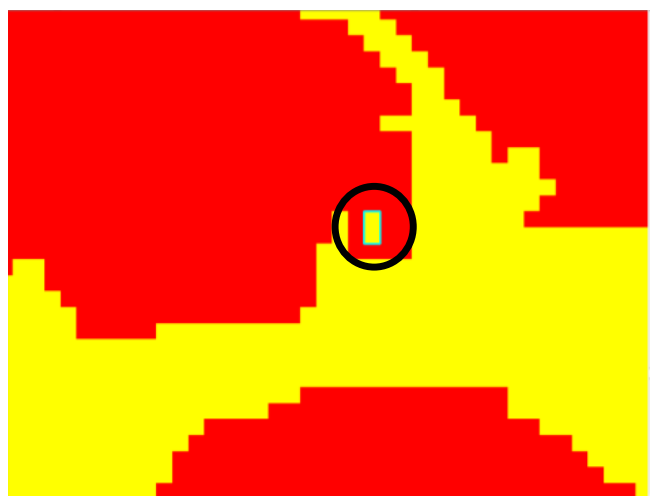

Figura 7 - Exemplo da edição manual de um polígono para o enquadramento na área mínima.

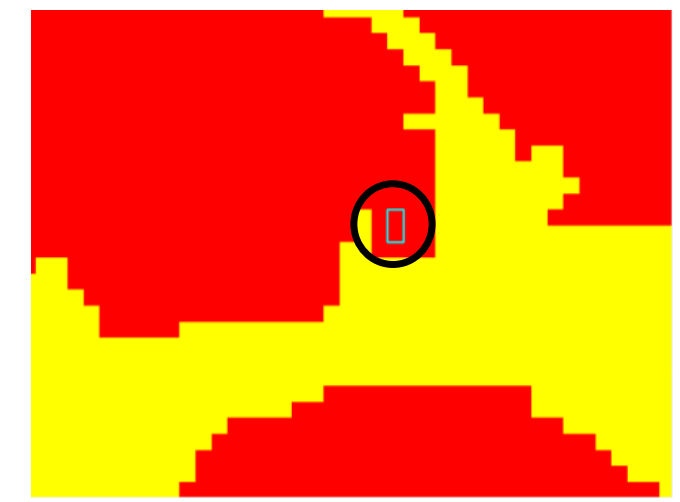

O mapeamento da detecção de mudanças no bairro do Camorim indica que do total da área do bairro, $19,12 \%$ sofreu com algum tipo de mudança em sua cobertura do solo e $80,87 \%$ não sofreu mudanças em sua cobertura. O mapa final pode ser observado na Fig.8. 


\section{Mapa de detecção de mudança do Camorim/JPA (2011-2015)}

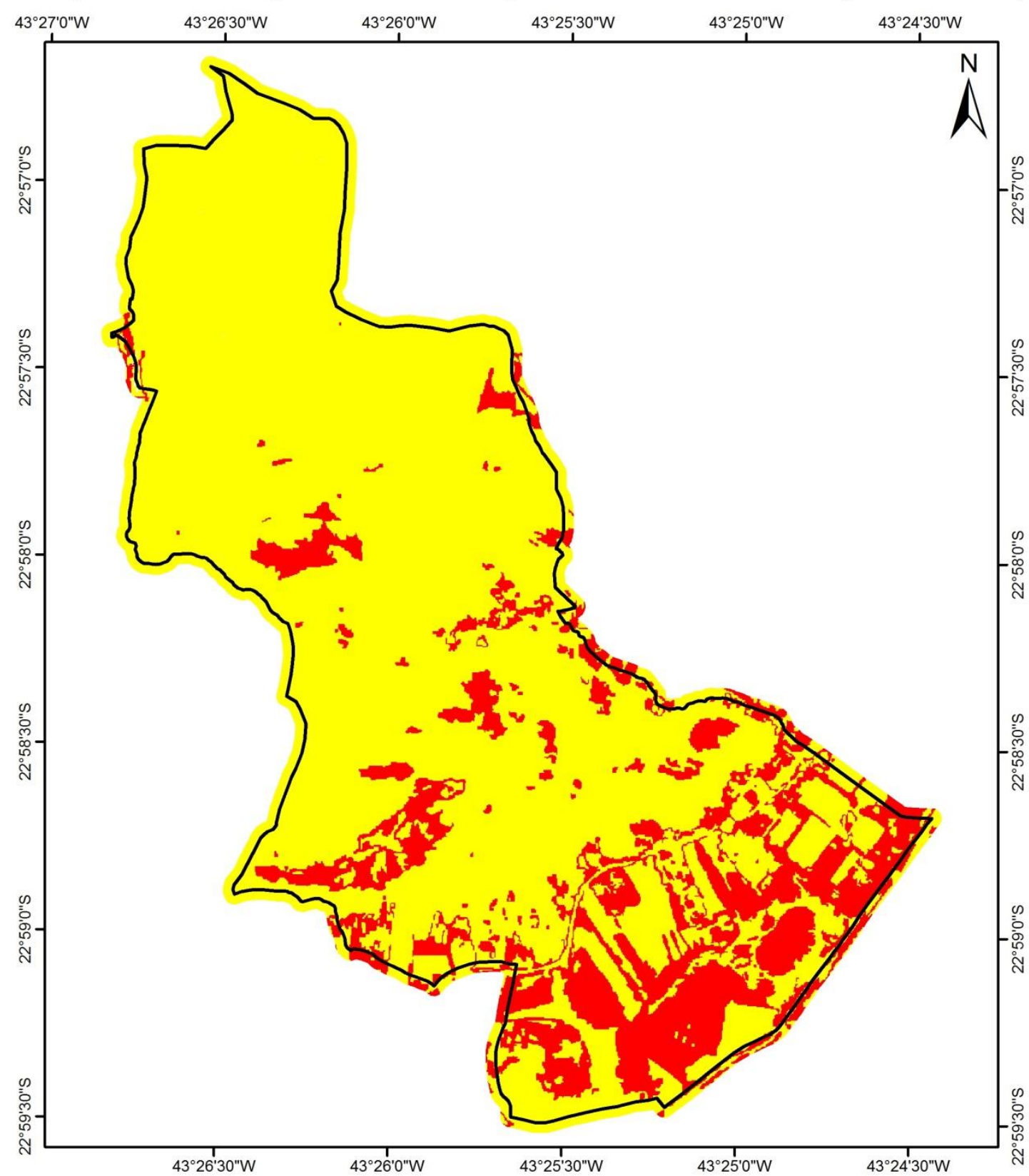

\section{Legenda}

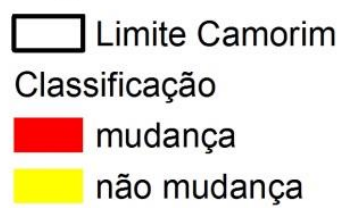

Sistema de Coordenadas Geográficas Projeção Cilíndrica Equirretangular Sistema Geodésico SIRGAS 2000

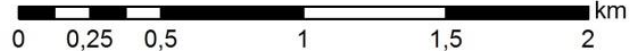

Figura 8 - Mapa de deteç̧ão de mudança no bairro do Camorim/JPA entre os anos de 2011 e 2015.

\subsection{VALIDAÇÃO}

Por último, após as etapas de pré-processamento, segmentação e classificação, fez-se necessário realizar a etapa de validação do mapa elaborado. Isto se deve ao fato de se ter conhecimento dos níveis de incerteza das classes trabalhadas. Nesse sentido, foi utilizado na presente pesquisa a matriz de confusão (Fig. 9) e o índice
Kappa, que tem por objetivo verificar o grau de concordância observada da classificação. Para isso, foi preciso anteriormente plotar pontos para validação. Esses pontos foram plotados de forma aleatória no software ArcGIS 10.1, através da ferramenta Create Random Points, sendo 30 pontos para cada classe. 


\begin{tabular}{|c|c|c|c|}
\hline Mapa & Mudou & Não Mudou & Soma das Linhas \\
\hline Mudou & 23 & 5 & 27 \\
\hline Não Mudou & 7 & 25 & 32 \\
\hline $\begin{array}{c}\text { Soma das } \\
\text { Colunas }\end{array}$ & 30 & 30 & 48 \\
\hline
\end{tabular}

Figura 9 - Matriz de confusão.

Pode-se apontar como uma diferença entre o índice de exatidão global, gerado pela matriz de confusão, e o índice Kappa, que o índice Kappa considera todos as áreas, inclusive aquelas que apresentem uma classificação indevida. Para o presente trabalho foi calculado o índice de exatidão global, a precisão de cada classe e o índice Kappa. Seus resultados foram de 0,80 para exatidão global, 0,77 de precisão na classe mudança, 0,83 de precisão para a classe não mudança; o valor de Kappa foi 0,60 . Esse resultado se enquadra dentro da qualidade de classificação boa (Tab. 1), segundo Rovedder (2007) para o índice Kappa.

Tabela 1 - Medida de concordância entre os dados observados

Fonte: Adaptado de Rovedder (2007)

\begin{tabular}{c|c}
\hline Kappa & Qualidade da Classificação \\
\hline$<0,00$ & Péssima \\
\hline $0,00-0,20$ & Ruim \\
\hline $0,21-0,40$ & Razoável \\
\hline $\mathbf{0 , 4 1 - 0 , 6 0}$ & Boa \\
\hline $0,61-0,80$ & Muito Boa \\
\hline $0,81-1,00$ & Excelente \\
\hline
\end{tabular}

\section{DISCUSSÃO E CONSIDERAÇÕES FINAIS}

No trabalho buscou-se realizar a detecção de mudanças através do Sensoriamento Remoto utilizando uma abordagem que vem se mostrando cada vez mais frequente em classificações baseadas em GEOBIA, que consiste na modelagem do conhecimento a partir das áreas de mudança e não mudança. Esta abordagem permite um significativo ganho de tempo de modelagem, visto que, supera a necessidade da realização de dois mapeamentos de cobertura para diferentes datas e a posterior comparação para que se torne possível à descoberta das áreas de mudança.

Alguns entraves metodológicos foram observados ao longo do processo de classificação, como por exemplo, a dificuldade em lidar com mudanças em regiões que já apresentassem níveis de influência antrópica elevada, como no caso da mudança de uma estrada de terra para canteiro de obras.

Outro aspecto visível que podemos destacar é a diferença na inclinação do sensor, tendo gerado áreas de sombra em regiões distintas nas duas imagens e dificultando o mapeamento em algumas regiões. Por serem imagens também provenientes de uma constelação composta por cinco satélites, diferenças na calibração destes sensores podem gerar respostas diferentes entre as duas datas. Diferenças estas que mesmo realizando a normalização entre as imagens podem ser difíceis de serem corrigidas e prejudicarem o processo de modelagem.

Assim, mesmo sabendo que a modelagem ainda pode ser aperfeiçoada para um melhor resultado na classificação, pode-se concluir que a detecção de mudanças para o bairro do Camorim foi satisfatória, tendo identificado com um bom nível de precisão as regiões que sofreram alterações entre os anos de 2011 e 2015.

\section{REFERÊNCIAS BIBLIOGRÁFICAS}

ABREU, M.A. (2006). Evolução urbana do Rio de Janeiro. Instituto Municipal de Urbanismo Pereira Passos (IPP), Rio de Janeiro. 147p.

ANTUNES, M.A.H. \& SIQUEIRA, J.C.S. 2013. Características das imagens RapidEye para mapeamento e monitoramento e agrícola e ambiental. In: Anais XVI Simpósio Brasileiro de Sensoriamento Remoto - SBSR. Foz do Iguaçu, PR, Brasil.

CENSO DEMOGRÁFICO 2010. Características da população e dos domicílios: resultados do universo. Rio de Janeiro: IBGE, 2011.

CHEN, G.; HAY, G.J.; CARVALHO, L.M.T.; WULDER, M.A. Object-based change detection. International Journal of Remote Sensing. V. 33, N. 14, p. $4434-4457,2012$.

DESCLÉE, B.; BOGAERT, P.; DEFOURNY, P. Forest change detection by statistical object-based method. Remote Sensing of Environment. V. 102, p. $1-11,2006$.

SCHNEIDER, A. Monitoring land cover change in urban and peri-urban areas using dense time stacks of Landsat satellite data and a data mining 
approach. Remote Sensing of Environment. V. 124, p. $689-704,2012$.

SINGH, A. Digital change detection techniques using remotely-sensed data. International Journal of Remote Sensing.V. 10, N. 6, p. 989 - 1003, 1989.

SONG, X.P.; SEXTON, J.O.; HUANG, C.; CHANNAN, S.; TOWNSHEND, J.R. Characterizing the magnitude, timing and duration of urban growth from time series of Landsat-based estimates of impervious cover. Remote Sensing of Environment. V. 175, p. 1 -13, 2016.

Instituto Pereira Passos/Prefeitura da Cidade do Rio de Janeiro (2003). Coleção estudas da Cidade - Notas Técnicas n 6 e 7: Jacarepaguá e Irajá. 34p.

Prefeitura da Cidade do Rio de Janeiro - Secretaria Municipal de Planejamento e Coordenação Geral Superintendência do Desenvolvimento da Barra da Tijuca - SUDEBAR. (1977). Plano Piloto da Baixada de Jacarepaguá e a Expansão Urbana da Cidade do Rio de Janeiro. 85p.

ROVEDDER, J. 2007. Validação da classificação orientada a objetos em imagens de satélite IKONOS II e a elaboração de indicadores ambientais georreferenciados no município de Torres, planície costeira do Rio Grande do Sul. Dissertação de Mestrado do PPGRS CEPSRM/UFRGS. Porto Alegre, RS, Brasil.

Sítios consultados:

Instituto Pereira Passos/Prefeitura da Cidade do Rio de Janeiro. Armazém de Dados. Disponível em: <www.armazemdedados.rio.rj.gov.br>. Acessado em: 21 de maio de 2012. 